\title{
Wallet Operation Evaluation System Using Deep Learning
}

\author{
Junichiro Yamawaki ${ }^{1}$, Yasunari Yoshitomi ${ }^{2}$, Masayoshi Tabuse ${ }^{2}$, Taro Asada ${ }^{2}$ \\ 1: SKY Co., Ltd.,3-4-30 Miyahara, Yodogawa-ku, Osaka 532-0003, Japan \\ 2: Graduate School of Life and Environmental Sciences, Kyoto Prefectural University, \\ 1-5 Nakaragi-cho, Shimogamo, Sakyo-ku, Kyoto 606-8522, Japan \\ E-mail:\{yoshitomi,tabuse\}@kpu.ac.jp,t_asada@mei.kpu.ac.jp \\ http://www2.kpu.ac.jp/ningen/infsys/English_index.html
}

\begin{abstract}
As the average age of Japan's population increases, as a method for investigating mild cognitive impairment (MCI), wallet operation evaluations have been receiving considerable attention recently. Herein, we propose a system for conducting wallet operation evaluations based on deep learning. In our system, the bills and coins extracted from a wallet are automatically scanned and recognized, which makes it possible to evaluate a person's ability to correctly select and extract the correct bills and coins from the wallet within a reasonable period.
\end{abstract}

Keywords: Mild cognitive impairment, Dementia, Wallet operation evaluations, Deep learning

\section{Introduction}

The average age of Japan's population is increasing, and this trend is expected to continue. As a result, the number of elderly persons suffering from dementia is growing rapidly, and it has become necessary to find ways to identify persons suffering from mild cognitive impairment $(\mathrm{MCI})^{1}$ in order to ensure they have proper care while suppressing the progression of the disease.

As a method for investigating MCI, which is one of the pre-stages of dementia, wallet operation evaluations have been receiving considerable attention recently. ${ }^{2}$ However, in the normal course of such testing, the test subject must be observed by an examiner who provides instructions on how the evaluation is conducted, observes the testing, and record the results. ${ }^{2}$

In contrast, this paper reports on an automatic evaluation system in which deep learning is used to conduct wallet operation evaluations automatically.

\section{Wallet Operation Evaluations}

During a conventional wallet operation evaluation, ${ }^{2}$ a subject is tested by an examiner who instructs him or her on how to proceed, observes the process, and then records the results. This process can be broken down into the following six operational units:

(1) The examiner hands the test subject a wallet containing five ( $¥ 1, ¥ 5, ¥ 10, ¥ 50$, and $¥ 100$ ) Japanese coins each, five $¥ 1,000$ Japanese bills, and one ( $¥ 2,000, ¥ 5,000$, and $¥ 10,000$ ) Japanese bills each, as partially shown in Fig. 1. (In the wallet, the total number of coins is 25 , while that of bills is eight.)

(2) Next, the test subject is shown a sheet of paper describing two trials for 10 seconds. In the first trial, he or she is instructed to extract $¥ 1,165$ from the wallet and place the money on the desk. The second trial is the same but the amount is $¥ 123$.

(3) The test subject is instructed to begin the trials described in (2) when the examiner presses the button on his or her stopwatch.

(4) The test subject performs the two tests and reports his/her completion as instructed by the examiner.

(5) After visually confirming that both operations have been completed and receiving the test subject's report, the examiner presses the stopwatch button and informs the test subject that the evaluation is over.

(6) The time required by the test subject to complete both the first operation and second operation is then recorded by the examiner. 


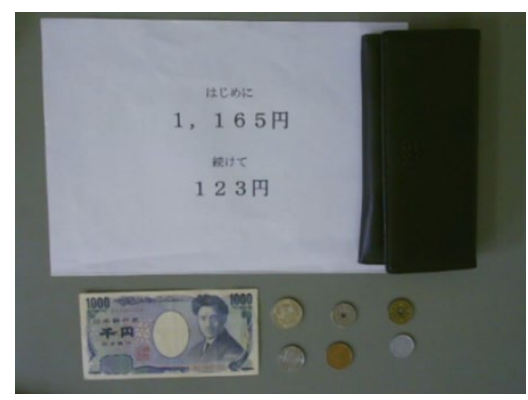

Fig. 1. Some of objects used in wallet operation evaluation.

\section{Proposed System and Method}

Our proposed system consists of two parts: (1) data preparation and deep learning and (2) the wallet operation evaluation module. Figure 2 shows the processing flow of the first part of this system, which consists of four processing units:

(1) Obtaining object image data

(2) Data augmentation

(3) Annotation

(4) Deep learning

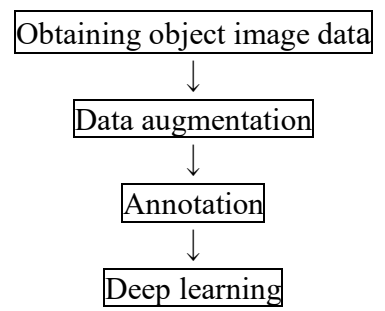

Fig. 2. Processing flow of data preparation and deep learning.

As objects to be recognized, we use $¥ 1, ¥ 5, ¥ 10$, $¥ 50, ¥ 100$, and $¥ 500$ Japanese coins and $¥ 1,000, ¥ 2,000$, $¥ 5,000$, and $¥ 10,000$ Japanese bills. For dataset assembly and recording the bills and coins during actual testing, we use a web camera set $40 \mathrm{~cm}$ above a desk plate to obtain object images, as shown in Fig. 3.

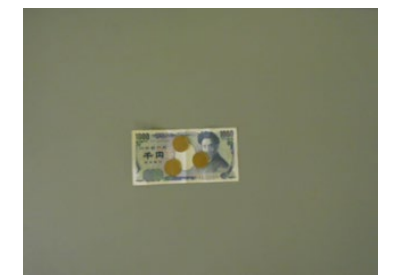

Fig. 3. Example of object image.

In this study, we use a region-based convolutional neural network (R-CNN) known as Faster R-CNN ${ }^{3}$ for deep learning and object recognition.

At the beginning of the evaluation, the same wallet containing the same coins and bills described in Section
2 is set in front of the test subject. The processing flow of the second part of the proposed system consists of the following 10 processing units:

(1)Japanese explanations of the evaluation process (three pages) are displayed sequentially on a personal computer (PC) screen, as shown in Fig. 4. The test subject indicates when he/she has finished reading each page by pressing the button connected to the PC.

(2)The PC then displays a 10-second message showing the two monetary amounts (first: $¥ 1,165$, second: $¥ 123$ ) the test subject will be requested to extract from the wallet.

(3)The evaluation begins when the test subject presses the button. At that point, the web camera and the system begin recording the elapsed time.

(4)The test subject extracts the appropriate bills and coins from the wallet and then presses the button again when he/she desires to report that an amount of money equal to $¥ 1,165$ has been placed on the desk plate as instructed

(5)An image showing the present state of the desk plate is then uploaded into the system.

(6)Next, the test subject proceeds to extract $¥ 123$ from the wallet and place it on the desk plate. Note that a condition of this test portion is that the test subject is instructed not to use the coins extracted for the $¥ 1,165$ test portion.

(7)The test subject then signifies the completion of that portion of the text by pressing the button again.

(8)An image showing the present state of the desk plate is uploaded into the system.

(9)Using object recognition, the total amount of money extracted from the wallet by the test subject, and the time required for both test segments are calculated by the proposed system.

(10)Finally, the time test and object recognition results are displayed on the PC screen, as shown in Fig. 5.
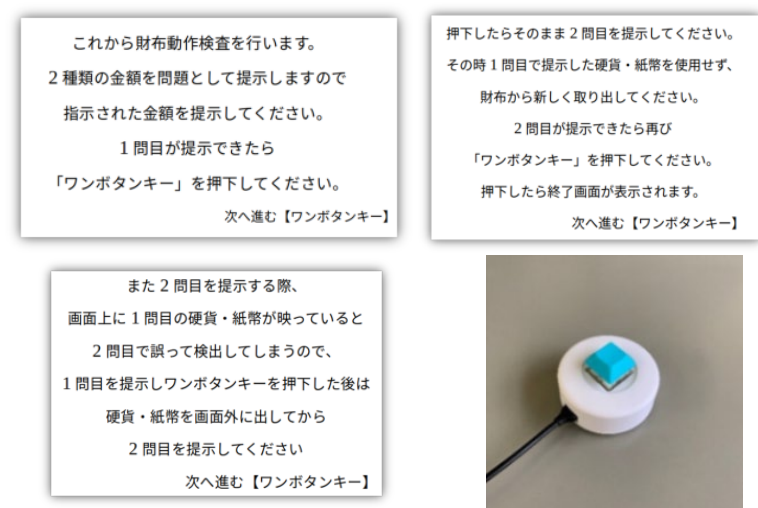

Fig. 4. Japanese language explanations of the evaluation process as displayed on the PC screen in order of upper left, upper right, and lower left. The bottom right photos show the button used for changing the display and recording elapsed time. 


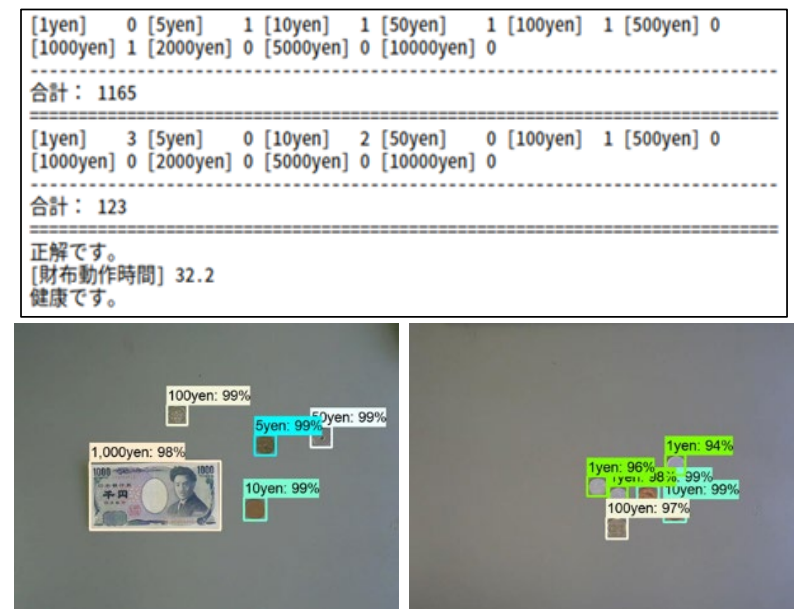

Fig. 5. Calculated evaluation results (upper) and object recognition results (lower left: $¥ 1,165$, lower right $¥ 123$ ).

\section{Experiment}

\subsection{Conditions}

The experiment was performed on a PC equipped with an Intel Core $17-6700 \mathrm{~K}$ central processing unit (CPU), a GeForce GTX 1080 graphics processing unit (GPU), and $32.3 \mathrm{~GB}$ of random access memory (RAM). The Ubuntu LTS18.04 64-bit operating system (OS) was installed on the PC and Python 3.7.3 was used as the development language. Jupyter Notebook, ${ }^{4}$ TensorFrow, ${ }^{5}$ ImageDataGenerator, ${ }^{6}$ labelImg, ${ }^{7}$ CUDA $10.0,{ }^{8} \mathrm{cuDNN}^{9}{ }^{9}$ and $\mathrm{OpenCV}^{10}$ were used as toolkits and libraries.

For moving image input, a stream web camera was used. ImageDataGenerator was used for data augmentation, while rotation, vertical shift, horizontal shift, expansion, shrink, and channel shift were used as processing. Next, labelImg was used for annotations.

For deep learning, the TFRcode ${ }^{11}$ for the faster_rcnn_resnet_101_coco model in TensorFlow ${ }^{11}$ was generated using a program obtained from Ref. 12. The mean average precision (MAP) obtained by the learning model used in the present study was 0.755 .

The resulting dataset contained 1,200 images showing the wallet and/or the Japanese bills and coins used in our experiment. Using ImageDataGenerator for data augmentation, 12,000 images were generated from the above 1,200 images. 10,000 of those images were used as learning data and the remaining 2,000 images were used for the evaluations. Figure 6 shows an example of data generated by augmentation.

To evaluate our proposed system, nine males (Subjects A to I) and one female (Subject J), all of whom are in their $20 \mathrm{~s}$, participated in our experiments.
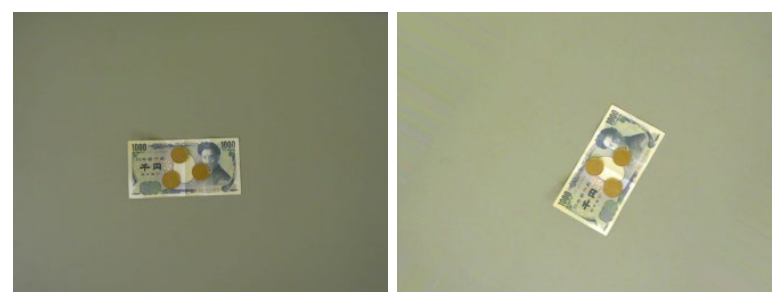

Fig. 6. Original image (left) and augmented image (right).

The experiments were performed under two conditions listed below:

Condition 1: with the conventional method $^{2}$ described in Section 2.

Condition 2: with the proposed system described in Section 3.

After the experiments, all of the test subjects were requested to select one of five answer-choices ([5] definitely yes, [4] yes, [3] no difference, [2] no, [1] definitely no) for each question in Table 1 in order to evaluate the proposed system.

Table 1. Questionnaire to evaluate the proposed system.

\begin{tabular}{cl}
\hline No. & \multicolumn{1}{c}{ Question } \\
\hline 1 & $\begin{array}{l}\text { Was the wallet operation test on condition } 2 \text { easier } \\
\text { than that on condition } 1 ?\end{array}$ \\
\hline 2 & $\begin{array}{l}\text { Did you feel less stress during the wallet operation } \\
\text { test on condition } 2 \text { than on condition } 1 ?\end{array}$ \\
\hline
\end{tabular}

\subsection{Results and discussions}

Table 2 shows the average time measured for the 10 subjects during these experiments. Here, it can be seen that the time required for the wallet operation evaluation using our proposed system was slightly longer than that of the conventional method. This might be caused by the additional button-pushing operations required during the process of our proposed method.

However, in both the conventional and proposed methods, none of the test subjects exceeded the limit time (120 s) that was assigned for the wallet operation evaluation in this experiment. This was thought to be because all subjects were in their $20 \mathrm{~s}$ and in good mental and physical condition.

Table 2. Comparison of the average time of wallet operation evaluation between the conventional and proposed methods.

\begin{tabular}{cc}
\hline & Average time (s) \\
\hline Conventional method & 32.2 \\
\hline Proposed method & 34.8 \\
\hline
\end{tabular}

(C) The 2021 International Conference on Artificial Life and Robotics (ICAROB2021), January 21 to 24, 2021 
Table 3 shows the average questionnaire score for the 10 subjects. Here, it can be seen that the proposed method was better than the conventional method from the viewpoints of ease of use and stress reduction.

For all of the objects on the desk plate, the average correct recognition rate by the proposed system was $80 \%$ for $¥ 1,165$ and $90 \%$ for $¥ 123$. Additionally, $¥ 100$ coins were sometimes misrecognized as $¥ 500$ coins (Fig. 7 ) and $¥ 1$ coins were sometimes misrecognized as $¥ 100$ coins, possibly due to light reflection. However, mistakes in both cases were rare.

As our future targets for improving our proposed system, we intend to perform the wallet operation evaluation with the participation of elderly persons who may have MCI or other forms of dementia. Moreover, we will reduce potential misrecognitions of the $¥ 1, ¥ 100$, and $¥ 500$ coins, primarily by making improvements to the lighting conditions and/or the learning dataset.

Table 3. Average questionnaire scores for 10 subjects.

\begin{tabular}{cc}
\hline Question No. & Average score \\
\hline 1 & 4.2 \\
\hline 2 & 4.1 \\
\hline
\end{tabular}

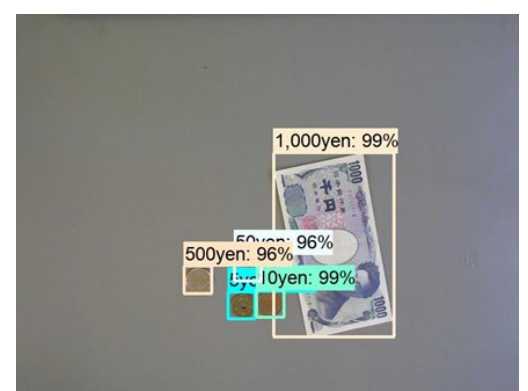

Fig. 7. Misrecognition of a $¥ 100$ coin that was identified as a $¥ 500$ coin.

\section{Conclusion}

As the number of elderly persons in Japan's super-aging society continues to increase, it is becoming increasingly important to find ways to identify persons suffering from MCI, which is one of the pre-stages of dementia, in order to ensure they receive proper care and make efforts to suppress the progress of the disease. As a method for investigating MCI, wallet operation evaluations have been receiving considerable attention recently.

Herein, we proposed a wallet operation evaluation system based on deep learning. In our system, a wallet containing bills and coins is supplied to the test subject, and his or her ability to extract bills and coins in the correct amounts is automatically recognized. The proposed system also determines whether the extractions from the wallet are made within a reasonable period.

\section{Acknowledgments}

The authors would like to thank Professor J. Narumoto of the Kyoto Prefectural University of Medicine for his valuable support and helpful advice during this research. We would also like to thank the test subjects of our experiments for their cooperation. This research was supported by COI STREAM of the Ministry of Education, Culture, Sports, Science and Technology (MEXT) of Japan.

\section{References}

1. B. Winblad, K. Palmer, M. Kivipelto, V. Jelic, L. Fratiglioni, et al., Mild cognitive impairment - beyond controversies, towards a consensus: report of the International Working Group on Mild Cognitive Impairment, J Int. Med., 256(3), 2004, pp. 240-246.

2. R. Senba, K. Kamijo, A. Kubo, and S. Murata, Comparison of wallet operations of community-dwelling elderly people by cognitive function category (in Japanese), Japanese J. Clinical Occupational Therapy, 3(1), 2016, pp. 5-9.

3. S. Ren, K. He, R. Girshick, and J. Sun, Faster R-CNN: towards real-time object detection with region proposal networks, in Advances in Neural Information Processing Systems 28 (NIPS 2015), eds C. Cortes and N. Lawrence and D. Lee and M. Sugiyama and R. Garnett, 2015.

4. Jupyter Notebook, https://jupyter.org/, Accessed 6 December 2020.

5. TensorFrow, https://github.com/tensorflow/, Accessed 6 December 2020.

6. ImageDataGenerator, http://keras.io/preprocessing/image/, Accessed 6 December 2020.

7. labelImg, https://github.com/tzutalin/labelImg, Accessed 6 December 2020.

8. CUDA, https://developer.nvidia.com/cuda-downloads, Accessed 6 December 2020.

9. cuDNN, https://developer.nvidia.com/cuDNN, Accessed 6 December 2020.

10. OpenCV, https://opencv.org/, Accessed 6 December 2020.

11. TensorFlow model zoo, https://github.com/tensorflow/models/blob/master/researc h/object_detection/g3doc/detection_model_zoo.md, Accessed 27 February, 2020.

12. https://github.com/EdjeElectronics/TensorFlow-ObjectDetection-API-Tutorial-Train-Multiple-ObjectsWindows-10, Accessed 27 February, 2020. 